there was no pigment. Both discs were white and the vessels much reduced in calibre. Fields, considerable loss in upper quadrant and whole central area. Pupils, moderate size, react normally. All deep reflexes very brisk. Flexor plantar responses. Liver and spleen not palpable. C.S.F. normal. Pr. 0.055 per cent.

Progress.-Certain subtle mental changes were present, for example, a certain facile contentment combined with a rather disproportionate emotional reaction. He was discharged unchanged.

( To be continued)

\title{
COLOUR IN PROTECTIVE NIGHT LIGHT
}

BY

\author{
The late C. E. FerReE and G. RAND
}

BALTIMORE, U.S.A.

IN the selection of a colour for a protective night light the eye factors as well as power to penetrate the external atmosphere should be taken into consideration-namely, the comparative sensitivity of the eye to coloured lights at very low intensities, visual acuity at low intensity, and the adaptation factors. Principally because of the eye factors deep red has recently been recommended as superior to the formerly widely-accepted dark blue light. The question is intricate and interesting and in its broader aspects is still an open one. It is extremely interesting in its relation to the visual functions.

Deep red light would hardly be acceptable as a night light for the illumination of many interiors, for example the lighting of hospitals. As a night light for hospitals we have used with considerable satisfaction light approximating the colour of a lowbrightness kerosene flame. This is not too unpleasant and is distinctly somnolent. The possibility of using heavily frosted, colourless bulbs "should not of course be ignored. In fact we believe that a comparison of test results will show in their favour. However, without the use of colour it is difficult to get light of sufficiently low intensity with the lamps that are available.

As to the suitability of blue as a colour for night-time protection, it should be kept in mind that dark blue is the least distinguishable from dark grey, and black of any of the shades of colour, and that the illumination of an interior with blue light by a suitable regulation of intensity and hue can be given a dim moonlight effect which differs little in colour from the exterior illumination on a moonlight night. Further, even if the brightness of the colours is 
selective to intensity and blue is seen as relatively lighter at low levels of intensity and with a dark-adapted eye than are the colours in the opposite half of the spectrum, this subjective effect can be compensated for by reducing by a corresponding amount the intensity of the blue light. Our thinking on this subject should, therefore, not be confused by too serious a consideration of the Purkinje phenomenon. Of the colours, red perhaps differs most in quality from black and for that reason red light stands out most vividly and strikingly from its background on a dark night. This is doubtless one of the reasons why red is chosen as a danger signal at night and might be offered as a very important reason for not using red as a black-out or protective light in war-time. On the other hand, in favour of red the photochromatic interval should be taken into account. That is, below the threshold of colour over a small range of intensity red light is not sensed as colourless as is the case with the other coloured lights. The question also should be raised of the relative visual acuities for blue and red light at low intensities.* Clearly the choice cannot be left to inference from 'scattered though related observations and experiments. It is a matter of direct test with as little as possible left to inference.

The problem is to select that colour or composition of light that will be of the greatest use in the discrimination of detail in nearby objects and will have the least visibility at a distance. The factors are the comparative visual acuities for different colours or compositions of light at a suitable low level of intensity, their power to penetrate the external atmosphere both clear and foggy, and their comparative visibilities in the dark at threshold intensities.

*Against the use of blue light the argument has been advanced that the eye is at a disadvantage in focusing blue light. One controversialist has even said that the eye cannot focus blue light. With respect to focusing the facts are: The emmetropic (normal) eye focuses parallel rays of blue light (i.e., objects at a distance of 20 feet or more) slightly in front of the retina. At some point nearer than 20 feet the rays diverge sufficiently to focus on the retina. This may be called the far point for blue light. For still nearer objects until the near point of vision is reached, the rays diverge progressively more and more, and require the lens of the eye to adjust (accommodate) for the respective distances. The near point for blue light is closer to the eye than for red, i.e., objects can be seen nearer the eye for blue than for red light. The focal point for parallel rays of red light for the emmetropic eye is behind the retina. For all distances, therefore, the eye has to accommodate for red light. The comparative situation, then, is $(a)$ that for blue light the emmetropic eye cannot focus for objects at a distance of 20 feet or greater, and for red light accommodation is required for objects at all distances; and (b) that the eye can see objects closer with blue light than with red light. Further, for uncorrected myopes (near-sighted) the eye with respect to focusing is better off with red light than with blue, and for uncorrected hyperopes (far-sighted) the converse is true-the eye is better off with blue light than with red. These conclusions based on the physical situation are borne out by the results which we have obtained on visual acuity and speed of vision with these two coloured lights for hyperopic, myopic and emmetropic eyes.

The following points may also be noted: (1) Because of the comparatively small amount of diffraction for blue light its effective power to give clear imaging for distances within the far point for blue is well recognized. This advantage is not 
had by deep red light. With respect to unclearness of imaging caused by diffraction, deep red light is the worst of the spectrum colours. (2) The disadvantage as to the co-ordination of accommodation and convergence is about equal for blue and red light. Complete co-ordination can be expected only for the wave-lengths in the mid region of the spectrum. The complete co-ordination of convergence and accommodation is, it may be noted, very important to comfort, efficiency and speed in the use of the eyes. (3) It has been argued that red light light-adapts the eye less than blue light and that, therefore, the eye retains its sensitivity to light better under red light than under blue and that correspondingly red light has an advantage over blue in the seeing of objects. At the very low intensities in question this factor cannot be of very great importance. In any event in the test which we are recommending later in the paper in which exactly the same intensities of light are used in determining the distances at which the different coloured lights can be seen as were used in giving equal visual acuities for these lights, this factor is fully taken into account. Furthermore, it does not seem that any difference in interference with subsequent trying to see in the dark could for lights of such low intensity be of any considerable importance. (4) Another argument that has been offered against blue light is that the colour field is wider for blue than for red. The thinking here seems to be confused by the fact that, in general, vision in the peripheral field is important in aviation, particularly in combat aviation where objects of considerable visibility are involved. In this problem, however, the situation is very different. In the first place the far periphery of the retina is not blind to colour. The limits of the colour fields are a matter of the amount or intensity of the coloured lights. In the second place the threshold of colour sensation is so much raised in the peripheral field that the use of peripheral vision for the detection of coloured light of threshold or near threshold intensity for central vision cannot be of significant importance. (5) Finally, as far as colour is concerned, a wider study by a test method that is direct and quantitative would probably show that the choice of coloured lights is by no means limited to red and blue. Both have important disadvantages. For example, when all are equalized in intensity or in both intensity and saturation, red and blue at any level of intensity as compared with most of the colours give very poor visual acuity or power to see objects. This is a very important point indeed. Except as an emergency expedient or a matter of feasibility it would seem somewhat strange if a choice between coloured lights were to be limited to the two that give such poor power to see objects, particularly when the distance at which the lights can be no longer seen depends so much more on their intensity than their colour.

Using spectrum lights we have shown that when equalized in brightness or photometric intensity, they give a wide range of difference in visual acuity, the acuity being the highest in the mid region of the spectrum, and that this range and variation from point to point are different for different levels of intensity of light. That is, visual acuity is selective both to wave-length or composition of light and to intensity of light. We found this to be true also when the lights were equalized both in brightness and saturation of colour. Some results of these determinations are given in the curves shown in Fig 1A. From an inspection of Fig. 1A it can be readily understood that the intensity of the coloured lights would have to be changed a great deal in relation to each other to give equal visual acuities, and that these changes of intensity would affect also greatly the distances at which they could be seen. The work was repeated using speed of discrimination, or speed of vision, as the test instead of visual acuity, the speed of the eye's reaction being considered an important factor in the decision as to the comparative merits of the lights in question. The results of these tests are given in the curves shown in Fig. 1B. Also using 
spectrum lights, equalized in energy, we have found that their visibility varies over a wide range, again being the highest in the mid region of the spectrum, and that the curve for visibility changes its shape for different levels of energy of light. That is, with respect to visibility, too, there is selectiveness to wavelength or composition of light, also to energy of light. These determinations were made by us with the light-adapted eye and under our direction with the dark-adapted eye. They constitute in effect a quantitative expression of the visibility changes that take place in

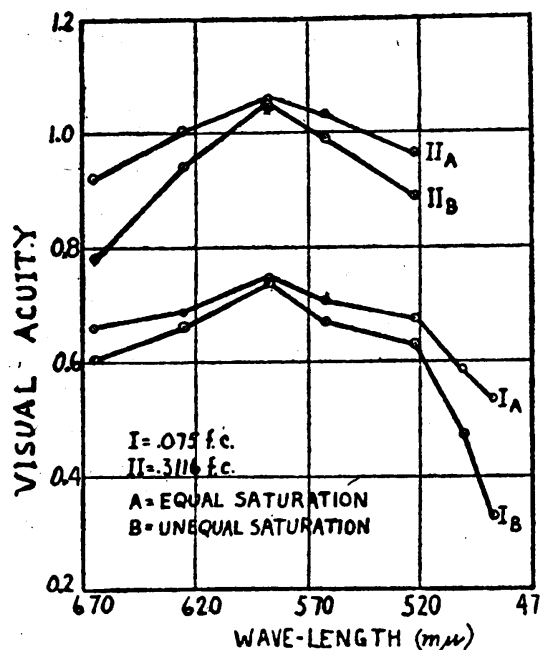

FIG. 1A. - The effect of change of wavelength of light on visual acuity at 0.075 and 0.3116 foot-candles, intensities $I$ and II. Unequal and equal saturation series (3 observers). *

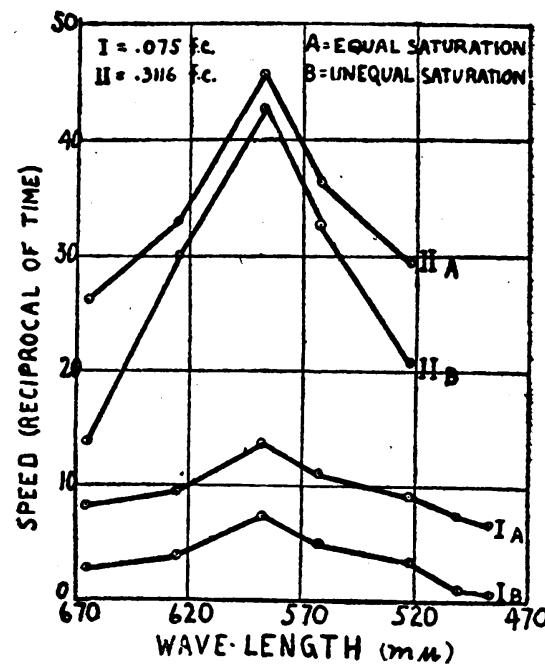

FiG. 1B.-The effect of change of wave-length of light on speed of discrimination at 0.075 and 0.3116 foot-candles, intensities I and II. Unequal and equal saturation series (3 observers). *

It may seem strange that when the different spectrum lights are themselves made equally visible there should be such a difference in the visibility of the details illuminated by these lights. In explanation, the following points may be noted: a difference in the clearness of the images formed, a possible difference in the retina's power to make fine space discriminations for the different wave-lengths, and the sensation difference between the black test-object and the coloured background. The complete picture of the eye's selectiveness of response to wave-length includes, then, definitely clearness of imaging, light and colour sensation both as to quality and amount, and possibly the power of the retina to discriminate space for the different wave-lengths of light.

*In the test for both visual acuity and speed of discrimination the distance of the observer from the test-object was 20 feet. Because of poor focusing at 20 feet this distance was of course unfavourable for the colours at the blue end of the spectrum. Another factor in the poor results obtained for blue light was the small quality or sensation difference between the black test-object and its blue background. 
what is commonly known as the Purkinje phenomenon. The results are too numerous and too complicated to warrant space being given to them here. It will be sufficient perhaps to say that the results for both visual acuity and visibility render it absolutely clear that in any determination of the best light to use for wartime protection, the tests for visual acuity and visibility must both be made and under exactly the same conditions with respect to the significant factors which influence each, namely intensity of light and colour or composition of light, and with a dark-adapted eye. This can be most certainly accomplished by using the same illuminator and the same setting of its intensity control for testing the comparative distances at which the lights can be seen as was used in making the test for the comparative acuities. That is, the exact source of the illumination in the first case is made the testobject in the second case. In this way all difficulties and sources of error in the duplication of conditions in the two items of the test are completely eliminated, a very important one of which is that of securing equal intensities through photometering lights differing in colour.

In order to make the test entirely direct, as should be the case, the intensity control of the illuminator should be set to give equal visual acuities at a suitably low level of intensity for each of the lights to be tested and the illuminator so adjusted be used, as noted above, as the test-object for the determination of the distance at which each light can be no longer seen. For still closer correspondence with the conditions in actual use, the size of the testobject, the distance of the testee and the distance of the illuminator from the test-object should be chosen so that the intensities which are required to give equal visual acuities are somewhat akin to the minimum level that should be had in seeing one's way about in the dark. Perhaps 20 feet could be used as the distance of both the testee and the illuminator from the test-object.* In our recommendation, however, full latitude is left on this point. In this way of making the test it should be clearly kept in mind that the size of the test-object, the distance of the testee and the distance of the illuminator from the test-object are kept constant, and the intensity

*It is doubtless better to use also for both the testee and the illuminator a distance which is representative of near vision, for example, a distance within the far point for blue light, and to base conclusions on the results of the tests for both distances. Unless results are obtained for distances that are representative of both far vision (20 feet or more) and near vision, it does not seem that a general conclusion can be safely drawn as to the merits of the lights in question. Further, objects and backgrounds present surfaces that have a wide variety of reflecting power. In order better to take into account the relationship of object to background as a factor in power to see objects, the intensities required to give equal visual acuities for the above-noted distances could, if thought best, be determined with both a black test-object on a white background and a white test-object on a black background, and these intensities also be used in determining the distances at which the lights can be no longer seen. 
of illumination is varied until the test-object chosen can just be discriminated. Another possibility is to adjust the intensities of light in the illuminator such that the distance at which each coloured light disappears is equal, and with this adjustment in case of each coloured light to determine the comparative acuities. The difficulty with this method would be in the equipment that would be required to vary in continuous change the size or visual angle of the critical detail in any test-object that would be suitable for the purpose without changing the distance of the testee from the test-object.

The distance test should of course be made under more than one atmospheric condition-clear, foggy, etc. Inasmuch as changing the distance in making the test is the most troublesome and timeconsuming part of the test; it may be found convenient to use as many illuminators as there are coloured lights to be tested. This would enable the chànges of distance to be made in a continuous series and would guarantee that the tests for the different lights are made under the same atmospheric condition.

Obviously the equipment used for making the test is very important to its success and its precision. The illuminator must be such, for example, that the changes of intensity of light can be made in continuous series without changing the colour or composition of the light, or the size, shape and location of the illuminated area. These requirements rule out the use of a rheostat or change of distance as means of changing the intensity, the rheostat in particular being unsuitable because of the changes which it causes in the colour and composition of the light. The further requirement that the illuminator be made the test-object in the distance test would make inconvenient the use of a projection system with an iris diaphragm control such, for example, as is used in our Visual Acuity Projector.* The instrument we would recommend as being the most suitable in all respects is shown in Fig. 2. It may be called a variable illuminator.

The purpose of this instrument is to provide a means of varying the intensity of the illumination in continuous change from zero to any intensity that is wanted, without change in the colour or composition of the light or in the size, shape or position of the illuminated area, and, if desired, to provide a means of correcting artificial light to light of daylight quality. The essential features of the instrument are a source of light of adequate intensity to give the range needed, a housing of suitable size and shape, a mechanical means. or specially designed shutter for varying the intensity of the light, and a diffusing plate or other diffusing means

\footnotetext{
* Ferree, C. E. and Rand, G.-Testing Fitness for Night Flying. Visual Acuity. Arch. of Ophthal., Vol. XX, pp. 58-79., 1938.
} 
both to eliminate completely the shadows which would otberwise be produced by the shutter and to give an evenly distributed and well-diffused illumination of the test surface.

The means of varying intensity consists of four vanes which extend across the opening of the housing in such relation to each other that when their flat surfaces are parallel to the beam of light the maximum amount of light passes through the opening, and when they are rotated to a position at right angles to the beam the light changes in continuous series from-full intensity to zero. In changing the intensity the vanes are so actuated as to cause contiguous vanes to turn in opposite directions. This insures that there will be no shift in the position of the illuminated area and no change in its size and shape. When the vanes all move in the same direction, as is the case with Venetian blinds, all these changes take place. In various models of the instrument different means of actuating the vanes have been employed. 'In one model split gears were mounted on the ends of the axle pins of the vanes outside the housing, so adjusted as to take up all slack and backlash. In another a simple means has been devised which lies within the housing. It also is entirely free from slack and backlash. This is in the form of a thin plate with a central longitudinal slot through which pass the axle pins at the ends of the vanes. This slot permits the plate to travel back and forth as the vanes turn. Above and below it in alternate sequences are short vertical slots which engage pins suitably positioned at the ends of the vanes. To the end of the axle of one of the vanes is attached a knob. When this knob is turned the contiguous vanes rotate in opposite directions as is desired.

In order that no shadow shall be cast by the vanes in any position in their rotation a plate of Belgian flashed opal glass is mounted directly in front of them, between them and the surface to be illuminated. In addition to eliminating completely all shadows on the illuminated surface, the provision secures exceptionally good diffusion of light. Behind the plate may be placed the colour filter that is needed for obtaining the colour selected to be tested.

While the flashed opal glass positioned directly: in front of the vanes is sufficient to remove all trace of shadow from any surface to be illuminated at a distance of 5 inches or more, when looked at directly it is not itself seen as uniformly bright. Therefore, it could not be used as the test-object in making the distance test. In order to provide an evenly illuminated and evenly bright surface that can be used as the test-object in making the distance test, the construction of the housing is extended 5 inches beyond this plate of flashed opal glass and in its opening is mounted a second plate of flashed opal glass. In Fig. 2 the aperture shown, purposely made to suit a wide variety of conditions, may be larger 


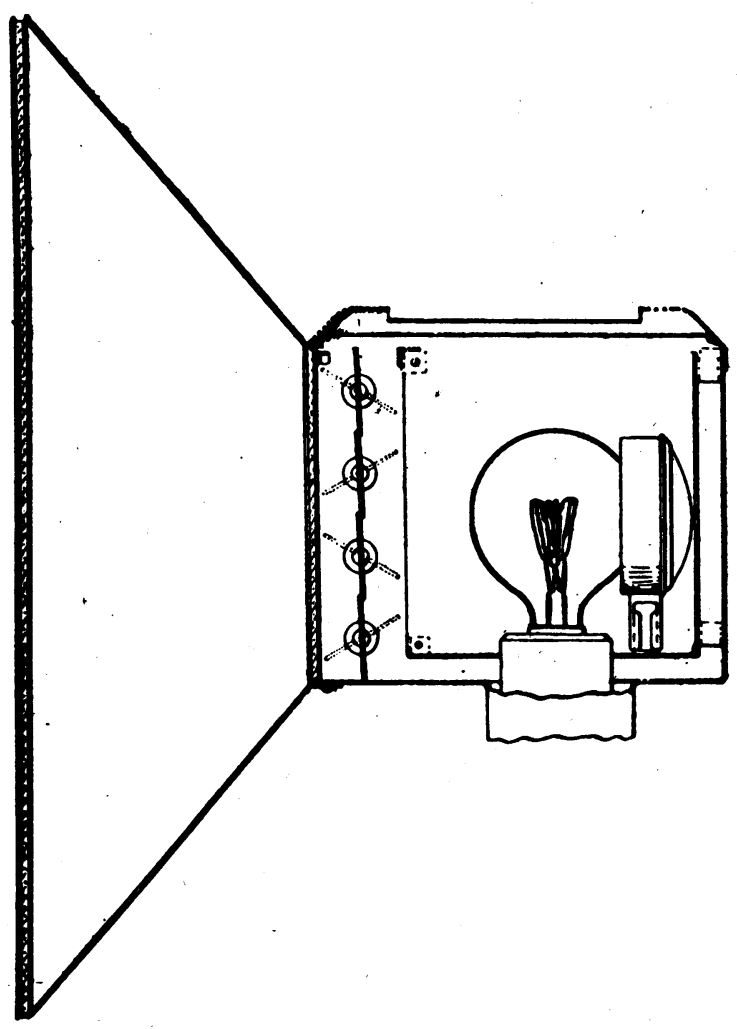

FIG. 2.-The variable illuminator, vertical section. Vanes in closed position indicated by solid lines, partly open by dotted lines.

than is wanted in making the test. It can be given any size and shape that is desired by the use of suitable diaphragms. In making the distance test, for example, some may not find it convenient or even possible to use great distances, in which case the aperture can be made small, but always of the same size and shape for the different colours. In the construction of this extension we have felt that for many reasons latitude with. respect to size and shape of this aperture was a desirable provision. The instrument so modified is used in making both the equalization of the visual acuities and the distance test. The front plate, then, of the instrument that is used in making the equalization of the visual acuities becomes the test-object or test surface for making the distance test, the intensity control having exactly the same setting for both tests.

As the most suitable test-object for making the equalization of the visual acuities we recommend the double-broken circle, the two 
breaks in which, $90^{\circ}$ apart, subtend each a visual angle of $1 \mathrm{~min}$. This type of test-object has been made into a chart which we have called the Double-broken Circle Visual Acuity and Astigmatism Test Chart (Fig. 3). Besides providing a more nearly absolute means of testing and rating vision, this chart comprises nineteen

\section{TEST CHART-SNELLEN RATING} reves ano enso

200

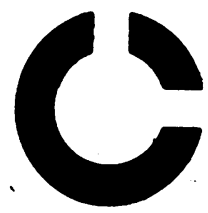

375

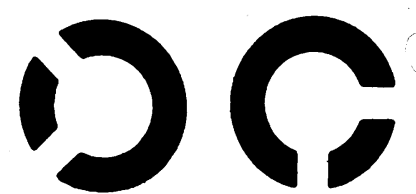

${ }^{150}$

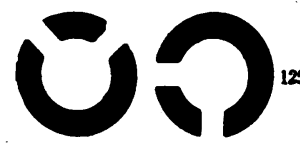

100

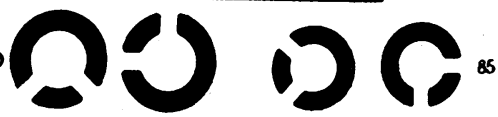

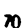

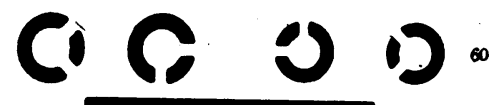

so

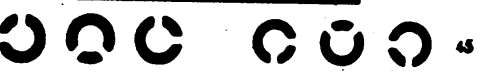

- UCC D o a

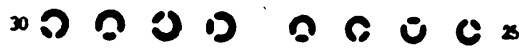

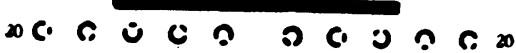

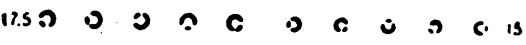

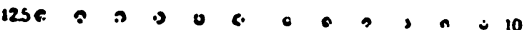

Fig. 3-The double-broken circle test chart.

200

(a)
as to size of critical detail; details to be discriminated (breaks in circle) simple and easy to grade in size and construct accurately; same type of task set by objects in different lines and equal task by all objects in same line-accurate rating scale readily possible; objective check on judgment; high sensitivity and close reproducibility of result; test objects difficult to memorize, also suitable for children and illiterates; method of scoring absolute in type, namely in terms of number of meridians in which critical detail can be discriminated; score shows whether astigmatism is present and indicates its approximate location. Capital-letter charts have none of these features. 
sizes of test-object, each size being presentta in enuugn positions to test and accurately measure visual acuity in eight representative meridians $-0.45^{\circ}, 90^{\circ}$, etc. This number of sizes, ten more than is ordinarily the case, renders the chart of especial value for selecting the size of test-object that is most suitable for making the present test. If desired, a single test-object of the size chosen can be used mounted on a rotatable dial with a remote control operated electromagnetically. The control we have devised for this purpose permits of the openings to be turned into and held in the eight representative meridians just noted- $0.45^{\circ}, 90^{\circ}$, etc.

The test we have recommended has the advantage of being both direct and quantitative, and the equipment of being, so far as we know, the most convenient and best suited to the purpose of any that has yet been devised.

\section{Black-out Lighting in the Home}

The variable illuminator has also been made in the form of a lamp with the opening turned downwards or obliquely downwards. With its control of intensity by means of the rotatable shutter, the light can be varied in continuous change from full to extinction, thus making it possible by using it as a night light to obtain a complete black-out or as near a complete black-out as may be desired. In this lamp any colour, type or wattage of bulb can be used, even the small neon night bulb. Suitably equipped, this lamp should serve a useful purpose in protective night lighting even to the total extinction of light for a complete black-out.

In this connection. we wish to point out again the infeasibility of the ordinary black-out devices as an all-the-year round means of securing a complete black-out: In our southern cities the thought of obtaining a complete black-out of a fully lighted room on a hot summer night for any considerable length of time by means of curtaining or similar devices alone, is untenable and intolerable. We say this after more than 30 years of experience in light-proofing rooms and ventilating light-proofed rooms. The light-proofing of rooms as an all-year-round project for the black-out without air conditioning, forced ventilation or a provision for natural ventilation with light-proofed devices seems to us not to be worthy of very serious consideration for the hot seasons of the year in hot climates. The black-out in the home is a very serious problem in this respect, and the home is the place where the greater part of our population stays at night. Very few homes are provided with air conditioning, forced ventilation or light-proofed means of natural ventilation. The kind of black-out that is used in England, for example, may not be suitable for use in summer in the southern portion of the United States. Again, what is feasible in 
winter-time will not do for the summer months. In short, it would appear that the problem of the black-out should be attacked from two sides-the conventional light-proofing devices, and lighting. The attack from the side of lighting can be made along two lines -colour and composition of light, and intensity and direction of light. For the control of intensity of light a means for producing a finely graded and continuous change down to extinction is desirable and in some cases essential. Our strong feeling of need for this twofold attack on the problem of the black-out and protective night lighting is one of the incentives that has caused us to take up the subject of lighting in relation to the black-out. Surely it is not as dangerous to have the amount of light that is needed just to be able to see to get around in one room of a house with its sheltering roof and walls as it is to have enough light to see to get around out of doors in the open; and in any prolonged black-out a certain number of people have to be able to see to get around out of doors.

\section{ANNOTATION}

\section{Moorfields in the Blitz}

Little can the founder of Moorfields in 1804 have conceived the possibility that his offspring would, nearly 140 years later, suffer damage from enemy action in what he might have been pardoned for thinking would be civilized times. But so it was, and now Mr. Arthur Tarrant, the secretary of the hospital, has contributed an excellent account of the war over Moorfields which, published in the Medical Press and Circular of January 27, 1943, will be read with interest by all old Moorfields students.

On the outbreak of hostilities 82 beds were reserved for air raid and service casualties; next a śmall institution outside London was converted into a fifty-bed ophthalmic hospital, complete with operating theatre. The usual civilian operative work was performed here. Out-patient attendances in the City Road temporarily diminished at the outbreak of war, but numbers, in the absence of air raids, steadily increased. An air-raid shelter was made in the basement, and with the start of the London blitz a "definite night life" was undertaken.

Moorfields was badly damaged by blast on January 11, 1941. Repairs were put in hand at once and work went on. Incendiary bombs fell on the hospital on April 15 and were extinguished by the engineers and fire watchers. The hospital would seem to have 